\title{
Blank size dependence of microstructure in radial-axial ring rolling of TA15 titanium alloy by macro-micro FE simulation
}

\author{
Lianggang Guo ${ }^{\mathrm{a}}$, Shuai Zhu, and He Yang \\ State Key Laboratory of Solidification Processing, School of Materials Science and Engineering, \\ Northwestern Polytechnical University, PO Box 542, Xi' an 710072, PR China
}

\begin{abstract}
The blank size, determining the deformation degree and the ratio of axial to radial feed amount of the radial-axial ring rolling process for TA15 titanium alloy, has a strong effect on the grain size and volume fraction of primary $\alpha$ phase, which determine the mechanical properties of the rolled ring. In this paper, a macro-micro 3D-FE model of the radial-axial ring rolling of TA15 titanium alloy has been developed under ABAQUS software. The ring blank transferring from heating furnace to rolling mill, rolling and cooling after rolling during the whole process are considered in the FE model. And multiple blanks with different rolling ratios and ratios of axial to radial feed amount are designed for a desired TA15 titanium alloy ring. Then, the effects of the blank size on the grain size and volume fraction of primary $\alpha$ phase in the rolled ring are investigated by comprehensive FE simulations. Finally, the optimum blank sizes are obtained by evaluating both the volume fraction of primary $\alpha$ phase and the distribution uniformities of grain size and volume fraction of primary $\alpha$ phase.
\end{abstract}

\section{Introduction}

TA15 titanium alloy rings, serving as key mechanical components, have been gaining increasing application in many industry fields, such as aircraft engine, rocket and marine engineering. Thus the microstructure of the ring parts must be strictly controlled to meet the demand of high performance. The volume fraction and distribution uniformity of primary $\alpha$ phase are the key microstructure parameters determining the mechanical properties of TA15 titanium alloy rings. The titanium alloy rings are mainly manufactured by the radial-axial ring rolling process. However, in this process, multiple blanks with different rolling ratios and ratios of axial to radial feed amount can be designed for the same desired ring part. The rolling ratio determines the deformation degree of the process, thus has a significant influence on the volume fraction and distribution of the primary $\alpha$ phase of the rolled TA15 titanium alloy rings. The ratio of axial to radial feed amount determines the compatibility of deformations in the radial and axial directions, consequently affecting the distribution of primary $\alpha$ phase. Thus, it is necessary to reveal the influence of blank size on the primary $\alpha$ phase in order to obtain high performance titanium alloy ring with preferable microstructure and properties.

\footnotetext{
${ }^{\text {a }}$ Corresponding author: glgglg66@nwpu.edu.cn
}

This is an Open Access article distributed under the terms of the Creative Commons Attribution License 4.0, which permits unrestricted use, distribution, and reproduction in any medium, provided the original work is properly cited. 


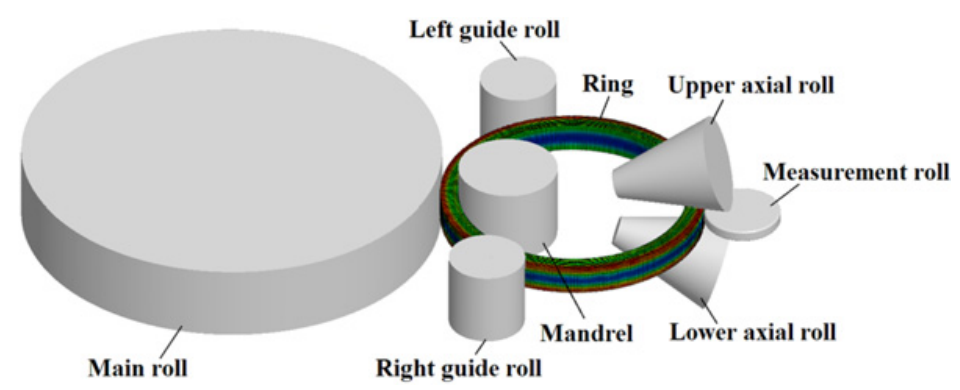

Figure 1. The macro-micro FE model for radial-axial ring rolling process.

Nowadays, some investigations concerning the microstructure evolution of titanium alloy during the ring rolling process have been carried out. Wang et al. [1] studied the evolution of $\beta$ phase in the ring rolling of Ti-6Al-4V alloy by implementing a microstructure evolution model into the FE model. Similarly, Zhu et al. [2] revealed the effects of deformation degree and initial forming temperature on the primary $\alpha$ phase of TA15 titanium alloy in hot ring rolling process. However, these works only involved the pure radial ring rolling process, which is a single-pass rolling technology. Its forming characteristics are different from that of the radial-axial ring rolling, and further the microstructure evolution may be different greatly. Neminathan et al. [3] introduced the production process of Ti-6Al-4V rings using the technology of the radial-axial ring rolling, and studied the final microstructure and performance of the rings. Recently, Zhu et al. [4,5] investigated the microstructure evolution of TA15 titanium alloy during the whole process of the radial-axial ring rolling, and revealed the effects of the initial forming temperature on the primary $\alpha$ phase. However, the above works were conducted under the condition that the blanks were the same. As well known, the blank size determines the deformation degree and compatibility of deformations in the radial and axial directions, and consequently has a crucial effect on the microstructure evolution during the process. Therefore, it is necessary to reveal the effects of blank size on the microstructure evolution in the process for the manufacturing of high quality titanium alloy rings and for the optimization of the blank size.

In the present work, we have developed a reliable macro-micro 3D-FE model of the whole radialaxial ring rolling process, including the ring blank transferring from heating furnace to rolling mill, rolling and cooling after rolling, under ABAQUS software. And multiple blanks with different sizes were designed for a desired TA15 titanium alloy ring. Then, the effects of the blank sizes on the primary $\alpha$ phase, grain size and their distribution of the rolled ring were investigated numerically, and the preferable blank sizes were obtained in consideration of the microstructure of the rolled ring. The results of this work can provide a guideline to the microstructure control and blank size design for the radial-axial ring rolling of titanium alloy.

\section{Macro-micro FE modelling for the whole process of radial-axial ring rolling of TA15 alloy}

In order to achieve the integrated prediction of deformation and microstruture of the rolling ring, a macro-micro FE model for radial-axial ring rolling of TA15 alloy has been established under the ABAQUS environment, as shown in Fig. 1. The key technologies used in the FE model are described as follows: (1) the rolls are treated as rigid bodies with analytical surfaces, and the motions of the rolls are real-time controlled based on in-process measurement using the VUAMP subroutine [6]; (2) the coupled thermo-mechanical hexahedron element with eight nodes is selected to mesh the ring, and an adaptive meshing technique is applied for maintaining a high-quality mesh; (3) three process stages including 
Table 1. Simulation conditions.

\begin{tabular}{|l|l|}
\hline Parameters & Value \\
\hline Radius of main roll $/ \mathrm{mm}$ & 550 \\
\hline Radius of mandrel $/ \mathrm{mm}$ & 130 \\
\hline Cone angle of axial roll $/{ }^{\circ}$ & 35 \\
\hline Temperature of rolls $/{ }^{\circ} \mathrm{C}$ & 150 \\
\hline Temperature of environment $/{ }^{\circ} \mathrm{C}$ & 25 \\
\hline Contact heat conductivity $/ \mathrm{W} /\left(\mathrm{m}^{2} \cdot{ }^{\circ} \mathrm{C}\right)$ & 4000 \\
\hline Convection coefficient $/ \mathrm{W} /\left(\mathrm{m}^{2} \cdot{ }^{\circ} \mathrm{C}\right)$ & 17.2 \\
\hline Heat radiation coefficient & 0.7 \\
\hline Friction coefficient & 0.3 \\
\hline
\end{tabular}

of ring blank transferring from heating furnace to rolling mill, rolling and cooling after rolling are simulated to realize the whole process modelling of radial-axial ring rolling; and (4) the microstructure model for TA15 alloy, developed and verified by Fan et al. [7], has been implemented into the FE model through VUSDFLD subroutine, thus the final microstructure of TA15 alloy rings can be predicted.

Based on the above FE model, the deformation and microstruture evolution during the whole process of radial-axial ring rolling of TA15 alloy can be predicted integratedly. And the FE modeling technologies and microstructure model of TA15 alloy used in present work have been verified to be reliable by Zhu et al. [4] and Fan et al. [7], respectively.

\section{Results and discussion}

\subsection{Simulation conditions and evaluation indices}

The ring blank size is determined by two key parameters [8]: rolling ratio $\lambda$ and ratio $k$ of axial to radial feed amounts. The rolling ratio $\lambda$ is defined as the ratio of cross-sectional area of blank to that of rolled ring. The ratio $k$ of axial to radial feed amounts determines the relative deformation amount in radial and axial directions of the ring. So multiple blanks with different $\lambda$ and $k$ can be designed for a desired ring. In this work, the sizes of the rolled ring are outer diameter $900 \mathrm{~mm}$, inner diameter $790 \mathrm{~mm}$, and height $120 \mathrm{~mm}$. For the sleeve-type rings, the feed amount in radial direction is usually larger than that in axial direction [6], and the inner diameter of the blank must be larger than the diameter of mandrel. Thus the ranges of $0<k<1$ and $1<\lambda<2.2$ can be obtained according to the design method of ring blank size in [8]. So $k=[0.1,0.3,0.5,0.7,0.9]$ and $\lambda=[1.3,1.5,1.7,1.9,2.1]$ are selected in this work. The $\beta$-transus temperature of TA15 alloy is $985^{\circ}$ and the heating temperature of blank is set to be $955^{\circ}$. The average feed rate of mandrel and angular velocity of main roll are selected to be $1.8 \mathrm{~mm} / \mathrm{s}$ and $2.1 \mathrm{rad} / \mathrm{s}$ respectively. The feed rate of upper axial roll is determined by the feed rate of mandrel and dimensions of blank and rolled ring [6]. The grain size and volume fraction of primary $\alpha$ phase of TA15 alloy at $955^{\circ}$ are $11.2 \mu \mathrm{m}$ and 0.207 , obtained by experiment, respectively. Some other simulation conditions are given in Table 1.

The preferable blank sizes are obtained in consideration of two requirements for the microstructure of the rolled ring: (1) the volume fraction of primary $\alpha$ phase in any zone of the rolled ring must be within the range of $20 \% \sim 40 \%$ due to the demand of high performance [3]; and (2) the distribution of both volume fraction and grain size of primary $\alpha$ phase should be homogeneous as far as possible. The standard deviation of the volume fraction $(S D F)$ and grain size $(S D D)$ of primary $\alpha$ phase are adopted 


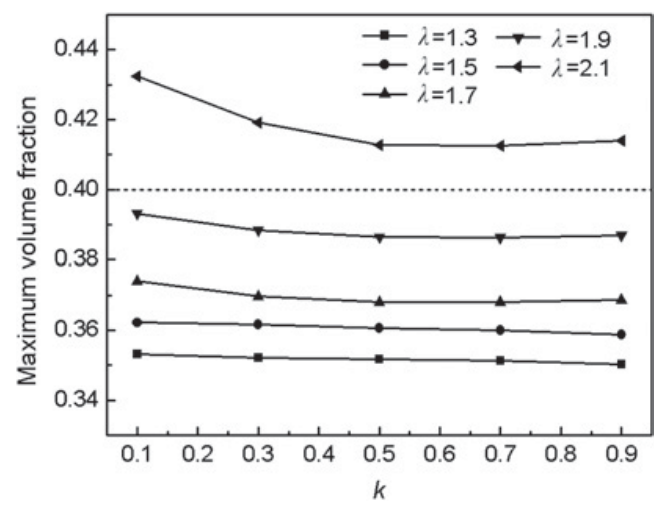

Figure 2. Maximum volume fraction of primary $\alpha$ phase under different blanks.

to describe the uniformities of the volume fraction and grain size, respectively.

$$
\begin{aligned}
& S D F=\sqrt{\sum_{i=1}^{N}\left(f_{i}-f_{a}\right)^{2} / N} \\
& S D D=\sqrt{\sum_{i=1}^{N}\left(d_{i}-d_{a}\right)^{2} / N}
\end{aligned}
$$

where $f_{i}$ is the volume fraction of primary $\alpha$ phase of node $i, N$ is the number of the nodes and $f_{a}$ is the average of volume fraction. $d_{i}$ is the grain size of node $i$, and $d_{a}$ is the average of grain size. The larger $S D F$ and $S D D$ are, the more nonuniform distributions of volume fraction and grain size of primary $\alpha$ phase are.

\subsection{Volume fraction of primary $\alpha$ phase}

The maximum volume fractions of primary $\alpha$ phase under different $k$ and $\lambda$ are shown in Fig. 2. With the increase of rolling ratio $\lambda$, the maximum volume fractions of primary $\alpha$ phase increase. The main reason is that: as rolling ratio $\lambda$ increasing, the grain sizes of primary $\alpha$ phase decrease due to the plastic strain becoming larger, and smaller grain sizes can lead to larger growth rates of primary $\alpha$ phase in cooling stage [4]. When rolling ratio $\lambda=2.1$, the maximum volume fractions of primary $\alpha$ phase are larger than $40 \%$. So, the rolling ratio of 2.1 is unqualified.

\subsection{Microstructure uniformity}

Under a certain rolling ratio $\lambda$, the grain size distribution of primary $\alpha$ phase becomes more nonuniform with the increase of the ratio $k$ of axial to radial feed amount, as shown in Fig. 3(a). The main reason is that the deformation amount in the axial direction becomes larger with $k$ increasing. So the plastic strain is mainly concentrated in the regions near the end surfaces of the ring. The larger plastic strain is, the smaller grain size of primary $\alpha$ phase is. Thus, the grain size in the regions near the end surfaces becomes smaller (Fig. 4), leading to the grain size distribution becoming more nonuniform. With the increase of $k$, the volume fraction distribution of primary $\alpha$ phase tends to get more uniform under $1.3 \leq \lambda \leq 1.7$, but that becomes more nonuniform when $\lambda=1.9$ and $k \geq 0.5$, as shown in Fig. 3(b). This is because, when $1.3 \leq \lambda \leq 1.7$, the grain size in the regions near the end surfaces becomes smaller with $k$ increasing, 

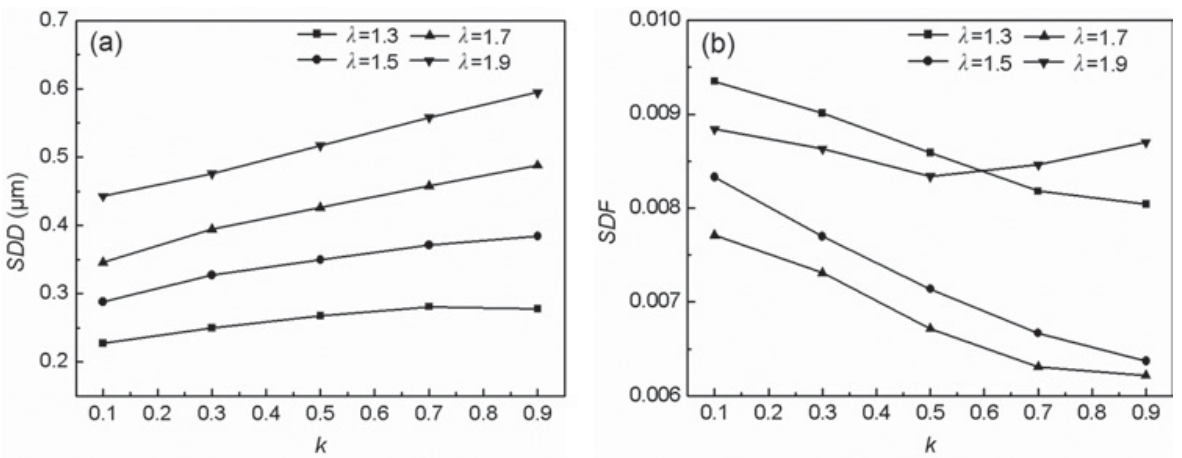

Figure 3. Uniformity of primary $\alpha$ phase distribution under different blanks: (a) grain size; (b) volume fraction.

(a)

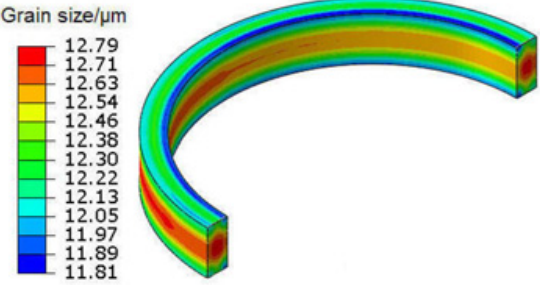

(b)

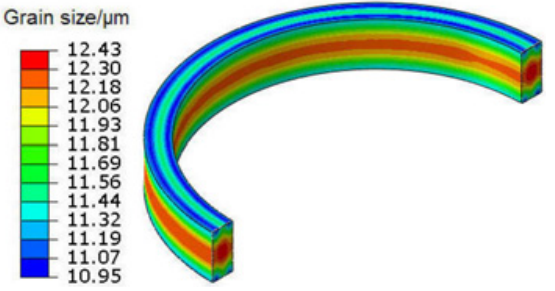

Figure 4. Grain size of primary $\alpha$ phase under different blanks: (a) $\lambda=1.3, k=0.3$; (b) $\lambda=1.7, k=0.5$.

(a)

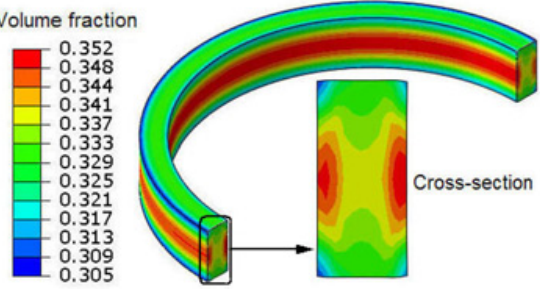

(b)

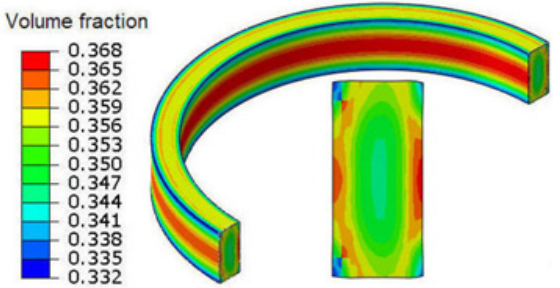

Figure 5. Volume fraction of primary $\alpha$ phase under different blanks: (a) $\lambda=1.3, k=0.3$; (b) $\lambda=1.7, k=0.5$.

and the growth rate of primary $\alpha$ phase with small grain sizes is large in the cooling stage. So the volume fractions of primary $\alpha$ phase near the end surfaces become larger (Fig. 5), leading to the volume fraction distribution of primary $\alpha$ phase tends to get more uniform. However, when $\lambda=1.9$ and $k \geq 0.5$, the deformation is very inhomogeneous at the rolling stage [6], resulting in the final volume fraction distributions becoming very nonuniform.

When $k$ is constant, with the increase of rolling ratio $\lambda$, the distribution uniformity of grain size of primary $\alpha$ phase becomes worse (Fig. 3(a)), while that of volume fraction gets better first and then worse (Fig. 3(b)). As the rolling ratio $\lambda$ increases, plastic strain distribution gets more nonuniform. So the distribution uniformity of grain size becomes worse. When $1.3 \leq \lambda \leq 1.7$, the grain sizes of primary $\alpha$ phase near the end surfaces decrease with $\lambda$ increasing after the rolling stage, which leads to the final volume fractions in these region becoming larger and further the volume fraction distribution of primary $\alpha$ phase getting more uniform on the cross-section of the ring (Fig. 5). However, when $\lambda=1.9$, the grain size distribution is very inhomogeneous after the rolling stage, thus the final distribution uniformity of volume fraction of primary $\alpha$ phase becomes worse. 


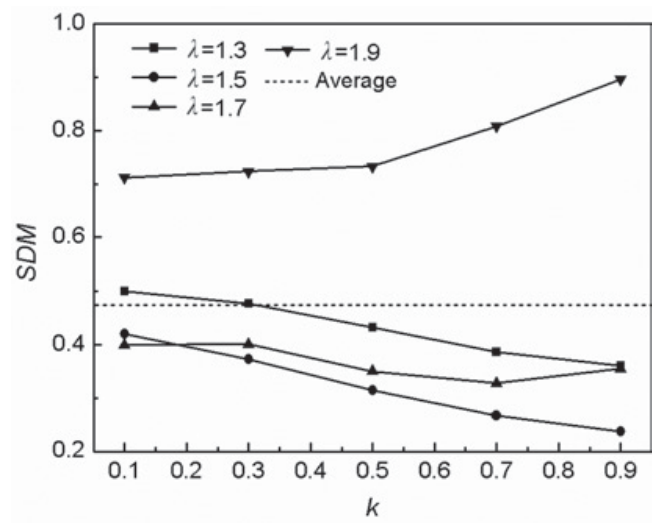

Figure 6. Microstructure uniformities under different blanks.

The distributions of both grain size and volume fraction of primary $\alpha$ phase have crucial effects on the mechanical properties of the TA15 alloy ring. Thus, the index SDM is defined for considering the grain size and volume fraction distributions comprehensively.

$$
S D M=0.5 \times \frac{S D F-S D F_{\min }}{S D F_{\max }-S D F_{\min }}+0.5 \times \frac{S D D-S D D_{\min }}{S D D_{\max }-S D D_{\min }}
$$

where $S D F_{\max }$ and $S D D_{\max }$ are the maximum $S D F$ and $S D D$ under the blanks with $\lambda=1.3 \sim 1.9$ and $k=0.1 \sim 0.9$, respectively; $S D F_{\min }$ and $S D D_{\min }$ are minimum $S D F$ and $S D D$, respectively. The smaller $S D M$ is, the more homogeneous microstructure is.

In the present work, the preferable blank should satisfies the condition that $S D M$ of the rolled ring must be smaller than the average of $S D M$ under the blanks with $\lambda=1.3 \sim 1.9$ and $k=0.1 \sim 0.9$. The microstructure uniformities under different blanks are shown in Fig. 6. It can be seen that the microstructure uniformities of many blanks with rolling ratio $\lambda=1.3 \sim 1.7$ are better. In radial-axial ring rolling process, when rolling ratio $\lambda$ being too small, the advantages of the process can not play its role and the material cost is usually large. Thus the optimum rolling ratio is suggested to be 1.7 in this work, and the optimum ratio of axial to radial feed amount is proposed to be $0.5 \sim 0.7$ due to the better microstructure uniformity, as shown in Fig. 6.

\section{Conclusions}

In the present work, the effects of the blank size on the primary $\alpha$ phase of TA15 titanium alloy ring are investigated numerically. The results obtained show that: (1) with the increase of rolling ratio, the maximum volume fraction of primary $\alpha$ phase in the rolled ring increases, and the grain size distribution of primary $\alpha$ phase becomes more nonuniform, while the distribution uniformity of volume fraction of primary $\alpha$ phase becomes better first and then worse; (2) with the increase of the ratio of axial to radial feed amount, the distribution uniformity of grain size becomes worse, while the distribution uniformity of volume fraction tends to being better; and (3) the optimum blanks are suggested to be the rolling ratio of 1.7 and the ratio of axial to radial feed amount of $0.5 \sim 0.7$ by comprehensively considering the volume fraction of primary $\alpha$ phase and the distributions of grain size and volume fraction of primary $\alpha$ phase. 


\section{ICNFT 2015}

The authors would like to thank the support of the National Natural Science Foundation of China (51175427, 51135007), Open fund of the State Key Laboratory of Materials Processing and Die \& Mould Technology, Huazhong University of Science and Technology(P2014-05) and the 111 Project (B08040).

\section{References}

[1] M. Wang, H. Yang, C. Zhang, L.G. Guo, Int. J. Adv. Manuf. Technol. 66, 1427 (2013)

[2] S. Zhu, H. Yang, L.G. Guo, R.J. Gu, Comp. Mater. Sci. 65, 221 (2012)

[3] P.V. Neminathan, M.S. Velpari, S.R. Ananda Rao, A.K. Gogia, Trans. Indian Inst. Met. 61, 355 (2008)

[4] S. Zhu, H. Yang, L.G. Guo, W.J. Di, Y. Fan, Chinese J. Aeronaut. 35, 3145 (2014) (in Chinese)

[5] S. Zhu, H. Yang, L.G. Guo, W.J. Di, Procedia Eng. 81, 274 (2014)

[6] S. Zhu, H. Yang, L.G. Guo, L.L. Hu, X.Q. Chen, Int. J. Adv. Manuf. Technol. 72, 57 (2014)

[7] X.G. Fan, H. Yang, P.F. Gao, J. Mater. Process. Technol. 214, 253 (2014)

[8] L.G. Guo, H. Yang, J.C. Jin, J. Mech. Eng. 46, 1 (2010) (in Chinese) 